\title{
INVESTIGATION OF THE DISSIPATION PROCESS IN ELECTROLYTIC CAPACITORS
}

\author{
LECONTE CATHEY and KEITH A. JOYNER \\ Department of Physics, University of South Carolina, Columbia, South Carolina 29208
}

(Received June 18, 1979)

\begin{abstract}
An experimental study of electrolytic capacitors was conducted, with emphasis on their thermal properties. The capacitors were subjected to charge-discharge cycles with various values of peak voltage. The observed power dissipated did not agree with that which would be expected if constant capacitance and constant effective series resistance (ESR) are assumed for the capacitors. In order to explain the discrepancy, the capacitance and ESR variations were measured with respect to voltage, temperature, and frequency. A predicted power dissipation was calculated for each capacitor; these dissipations compared favorably with those that were experimentally observed. In addition, voltage versus time profiles were measured for each unit and these were analyzed to determine the charge transport characteristics of the capacitors. The populations and mobilities of each type of charge carrier turned out to be quite similar from one capacitor sample to the next.
\end{abstract}

\section{INTRODUCTION}

In the electronics industry as well as most of the scientific community, it has been assumed that capacitors are linear devices or that the deviations from linearity are of no significance with regard to applications. ${ }^{1,2}$ An investigation into the thermal properties of aluminum electrolytic capacitors has revealed that there are important deviations from linearity which indicate that the characteristics of the capacitors should be carefully considered when specifications are made. These deviations are apparently due in large part to the fact that the charge in an electrolytic capacitor moves as if it were composed of several different types of charge carriers. These charge carriers move at different rates and show different populations.

A capacitor is said to be linear if the charge stored is proportional to the charging voltage and if the proportionally constant, $\mathrm{C}$, is not a function of voltage or temperature. It is also usually assumed that the effective series resistance, ESR, of a linear capacitor does not depend upon voltage, frequency or temperature. The ESR is that value of resistance which, when placed in series with an ideal capacitor, would give the observed dissipation factor and phase angle.

\section{EXPERIMENTAL DESCRIPTION}

The capacitors were placed in a calorimeter which consisted of a thermally isolated container constructed by fitting a Dewar flask with an insulating fiberglass lid. An insulating container was used so that the fractional change in temperature across the diameter of the capacitor would be as small as possible; that is, so that most of the temperature difference between the interior of the capacitor and ambient would appear across the Dewar flask. Electrical connection was made to the capacitors by large leads brought through the lid of the Dewar. Leads were also connected to a thermistor and thermocouple placed inside the flask. The thermocouple and thermistor were mounted on a heavy copper cylinder which contained the capacitor and a bath of mineral oil, thus providing essentially isothermal contact between the capacitor and the temperature sensors.

The power produced by the capacitor was determined, after the thermal equilibrium had been established, by measuring the difference between the temperature inside the Dewar and the ambient temperature. This temperature difference was converted into a dissipation power by comparison with a calibration curve for the Dewar and lid generated with an electrical heater in the Dewar. The capacitor was alternately charged and discharged by means of a rotary switch at the rate of $1.88 \mathrm{~Hz}$. The power supply for charging was regulated by a magnetic amplifier with a Sola constant voltage AC input. For each capacitor, five values of peak charging voltage were used and the power produced was determined for each. A plot of power versus peak charging voltage squared should have yielded a 
straight line. Instead, the dissipated power curved upward as peak voltage increased. This behavior was exhibited by each of the seven samples tested. The data were found to show a power law relationship;

$$
P=K^{*} V^{x}(x=2.45 \text { - 2.96). }
$$

It was suspected that the deviations from linearity were important in explaining these higher losses; so the dependence of the capacitance and ESR on voltage, temperature, and frequency was investigated. In order to determine the dependence of capacitance on temperature and voltage, the capacitors were placed in a hot box. Leads were brought out of the top of the box and the capacitance measured with a General Radio model 1617 capacitance bridge. This was done at temperatures corresponding to those equilibrium temperatures which the capacitors attained when being cycled in the calorimeter. An automated test facility at the Mepco/Electra Corporation plant in Irmo, South Carolina was used to determine the frequency dependence of the ESR above $60 \mathrm{~Hz}$ for these same temperatures. The ESR turned out to be essentially independent of bias voltage. However, since the capacitors were cycled at $1.88 \mathrm{~Hz}$, the ESR had to be determined down to about $2 \mathrm{~Hz}$. For this purpose, a differential phase measuring instrument was constructed with an oscilloscope as a detector. A "variable ESR" capacitor was constructed by placing a variable resistor in series with a capacitor having very low ESR. The "variable ESR" capacitor was adjusted at each frequency so that it had the same phase angle as the unknown capacitor. The ESR of the unknown could then be calculated if the capacitance of the unknown capacitor were determined at that frequency.

Since the capacitors being used were large and the frequencies were low, it soon became apparent that it would be very difficult to drive them at a signal level high enough for the capacitance to be determined with a conventional capacitance bridge. Since iron core inductors show a large frequency dependence in their induction at low frequencies, it was not feasible to determine the capacitance with a series resonant circuit. It was decided that the low frequency capacitance could be determined (or at least estimated) by measuring the charge carrying characteristics. By this method, it would be determined if the capacitance could be treated as a constant below $60 \mathrm{~Hz}$. In order to determine the populations and mobilities of the charge carriers, the capacitors were charged to about $12 \mathrm{~V}$ and then discharged through a $1000 \mathrm{ohm}$ precision resistor.

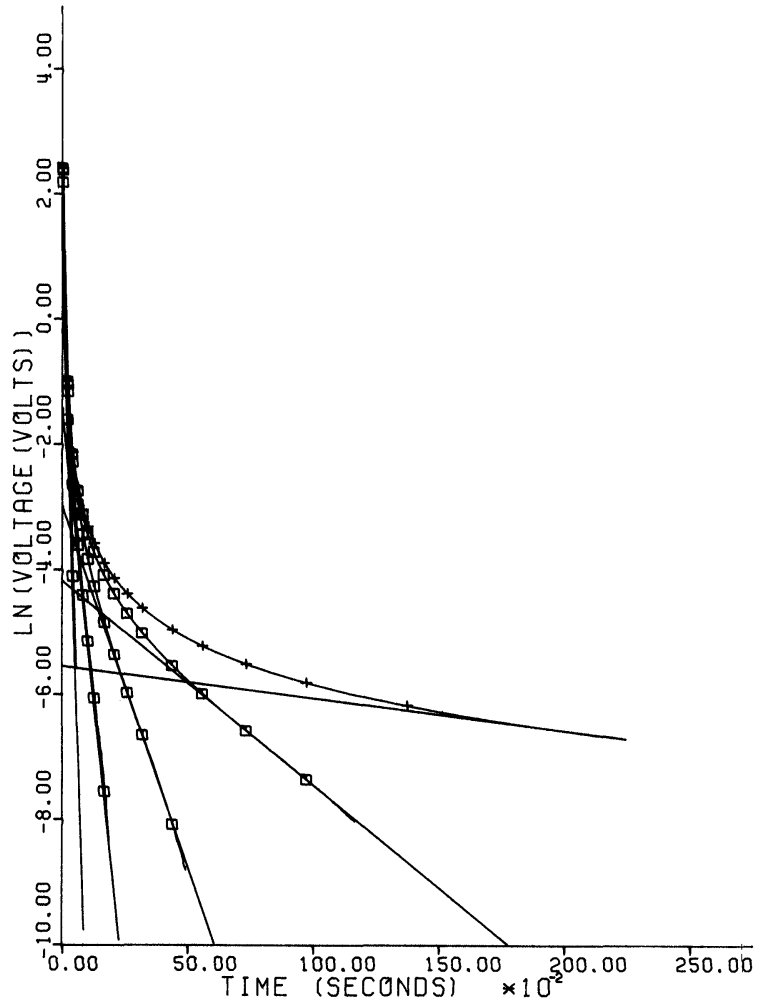

FIGURE 1 Natural logarithm of the voltage across capacitor number $3(+)$, the extrapolations $(-)$, and the differences $(\square)$ versus time. Every 20 th point is marked.

The voltage was measured as a function of time for periods of 18000 to $25000 \mathrm{sec}$. The logarithm of these voltages was plotted versus time to determine if the slow charge had an appreciable effect on the capacitance at $1.88 \mathrm{~Hz}$ and if the different capacitor samples had slow charge components with appreciably different populations or mobilities. The result for one of these samples is shown in Figure 1. In order to separate the different charge carrying components, the slope of the curves was determined by least squares fitting for the portion corresponding to the largest values of time. This straight line was extrapolated back toward zero time and the difference between this curve and the original data was taken in logarithmic fashion. This difference was plotted and the linear portion of the difference curve was extrapolated in similar fashion. By repeating this procedure several times for each sample, we were able to separate different types of charge carriers and determine whether the assumption of essentially constant capacitance below $60 \mathrm{~Hz}$ were valid. A summary of the results of these operations is given in Table I. 
TABLE I

Listed are slopes and intercepts of the extrapolations made from voltage versus time data. The values given are averages for all units tested

\begin{tabular}{llll}
\hline $\begin{array}{l}\text { Extrapolation } \\
\text { Number }\end{array}$ & $\begin{array}{l}\text { Logarithmic Slope } \\
\left.\operatorname{Ln}\left(V_{0}\right)-\operatorname{Ln}(V)\right) /\left(t_{0}-t\right)\end{array}$ & $\begin{array}{l}\text { Intercept } \\
\operatorname{Ln}(V)\end{array}$ & $\begin{array}{l}\text { Fraction of } \\
\text { Total Charge }\end{array}$ \\
\hline 1 & $-6.29 \times \operatorname{Exp}(-05)$ & -5.62 & 0.0003 \\
& $\pm 1.43 \times \operatorname{Exp}(-05)$ & \pm 0.37 & \\
2 & $-4.25 \times \operatorname{Exp}(-04)$ & -4.35 & 0.0011 \\
& $\pm 0.95 \times \operatorname{Exp}(-04)$ & \pm 0.40 & \\
3 & $-1.72 \times \operatorname{Exp}(-03)$ & -3.08 & 0.0039 \\
& $\pm 0.33 \times \operatorname{Exp}(-03)$ & \pm 0.21 & \\
4 & $-5.45 \times \operatorname{Exp}(-03)$ & -1.38 & 0.0214 \\
& $\pm 0.85 \times \operatorname{Exp}(-03)$ & \pm 0.18 & \\
5 & $-1.63 \times \operatorname{Exp}(-02)$ & 1.71 & 0.469 \\
& $\pm 0.24 \times \operatorname{Exp}(-02)$ & \pm 0.68 & \\
remaining & $-2.5 \times \operatorname{Exp}(-02)$ & 1.79 & 0.505 \\
fast charge & & & \\
\hline
\end{tabular}

\section{DISCUSSION}

Although the capacitors were rated at $0.039 \mathrm{~F}$ and $45 \mathrm{~V}$, they were charged to less than $28 \mathrm{~V}$ during these cycles. Dissipation power levels of $8 \mathrm{~W}$ were observed, however, and it seems likely that the units would have been destroyed it they had been operated near their rated voltages. An indication of the importance of this difference between the expected dissipation and that observed is given in Figure 2. At the highest voltage used, the observed losses were 2.4 times the 'expected' losses. The measurements revealed that the capacitance increased with increasing temperature and also increased with increasing voltage, as indicated in Figure 3 . The ESR was found to decrease slightly with increasing temperature and decrease as the frequency increased from $2 \mathrm{~Hz}$; see Figure 4. Since the ESR is not usually specified below $60 \mathrm{~Hz}$, this means that the losses rise as the frequency is reduced below $60 \mathrm{~Hz}$.

Since the voltage waveform applied across the capacitors varied approximately exponentially with time, it was necessary to analyze it into its frequency components so that each of them could be treated separately. This was done by Fourier analysis and the power dissipated was calculated for each component, taking into consideration the dependence of capacitance on voltage and temperature and the dependence of ESR on temperature and frequency. This analysis was done numerically by computer, using the measured values and no 'fitting' parameters. In order to calculate the losses at each frequency in the Fourier spectrum it was necessary to determine the capacitance at voltage increments along the charge-discharge curve for each different temperature and to determine the ESR for different temperatures and at different frequencies in the Fourier spectrum. The energy dissipated for each component in the Fourier spectrum could then be calculated and their contributions summed. For every sample except one, the computed dissipation agreed with that observed experimentally to within $47 \%$ in the worst case, and typically to within $25 \%$. The exception, sample \#1, was subjected to appreciably more abuse than the others, and its parameters had changed significantly between the time the dissipation was measured and the time the low frequency ESR was measured. It should be noted that these capacitor samples were not all from the same manufacturing run. In addition, these capacitors had considerably different life histories and there are differences in characteristics between samples which are ostensibly identical. In view of these facts, the correspondence between the tested units must be considered good.

The use of Fourier analysis implies that an assumption of frequency linearity or orthogonality of the various frequency components has been made. However, the entire analysis is predicated upon the assumption that the capacitors are nonlinear. A test was made to determine if the different frequencies were indeed orthogonal. A sine-wave frequency generator was set to approximately $2 \mathrm{~Hz}$ and used to drive a circuit which consisted of a resistor in series with either another resistor or one of the capacitor samples. The waveform across the second resistor and 




FIGURE 2 Measured values of power dissipated $(\bullet)$ versus the square of the peak charging voltage along with the expected dissipation based on the initial slope, which would correspond to a prediction based on specified ESR.

the capacitor were analyzed with an audio spectrum analyzer to see if the presence of the capacitor changed the frequency spectrum of the waveform. No change in the frequency spectrum was detected when the capacitor was connected into the circuit.

The dependence of capacitance on voltage and temperature and the dependence of ESR on frequency and temperature are believed to be due to the presence of 'slow charge' in the capacitor. ${ }^{3}$ This slow charge is thought to consist of different ion types or of electrolyte ions which have penetrated into the oxide film in submicroscopic channels or inclusions. In one of the units, the one which was discharged to the lowest voltage, an extra charge carrying species was found which did not appear in any of the other capacitors. This charge carrier has a mobility which is intermediate between the mobilities of two of the species of the other capacitors. The reasons for this are not clear at this time and it would be necessary to take data for a considerably longer discharge time before the characteristics of the charge carrying species could be given definitively. The principal purpose of the voltage discharge experiment was to determine if the assumption of essentially constant capacitance below $60 \mathrm{~Hz}$ were justified. Since there are two "fast" charge carriers and several more which were considerably "slower", it is safe to treat the capacitance as a constant below $60 \mathrm{~Hz}$. 




FIGURE 3 Capacitance in millifarads versus bias voltage for sample number 3 at the equilibrium temperatures.

\section{ACKNOWLEDGEMENTS}

The authors gratefully acknowledge the aid of Mr. K. L. Benson, Dr. J. S. Shaffer, Mr. J. E. Reeves and Mr. M. E. Fulmer of the Mepco/Electra Corporation, Irmo, South Carolina, for assistance in the use of their facilities and measuring instruments. We also thank the General Electric Capacitor Division for providing electrolytic capacitor samples.

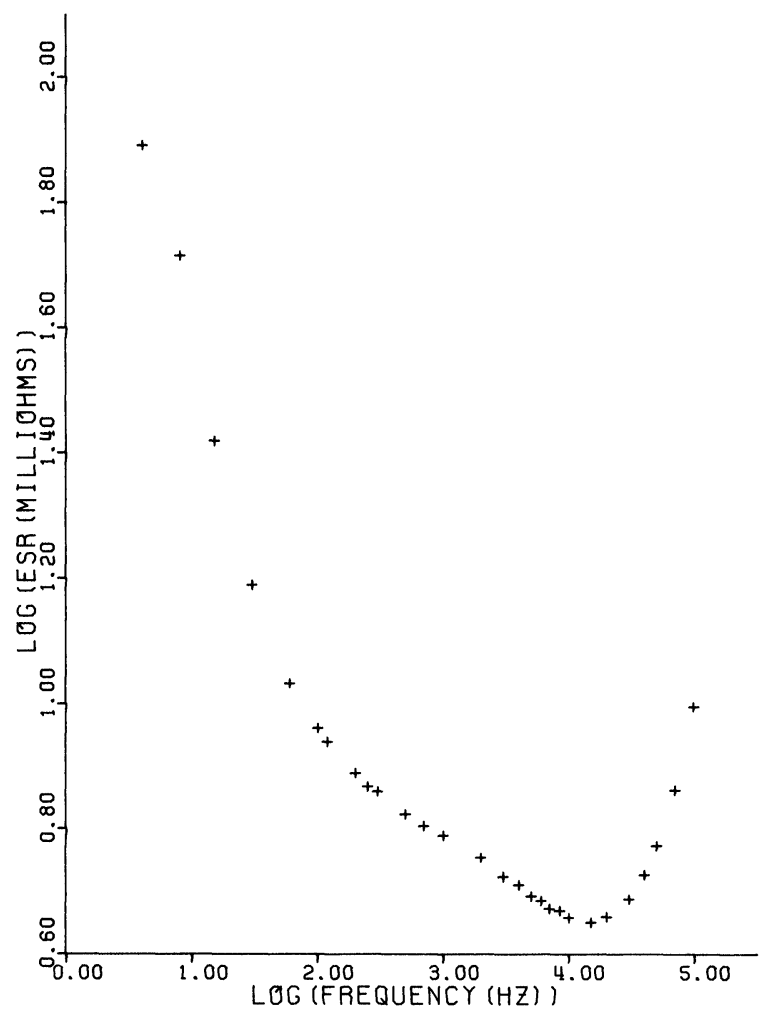

FIGURE 4 Logarithm of effective series resistance (ESR) versus logarithm of frequency for sample number 3 at $70.0^{\circ} \mathrm{C}$.

\section{REFERENCES}

1. E. Bowling, Electron. Ind. 31 June (1964).

2. F. G. Hayatee, Electrochem. Sci. Tech. 2109 (1975).

3. E. Von Schweidler, Ann. Physik 24711 (1907). 

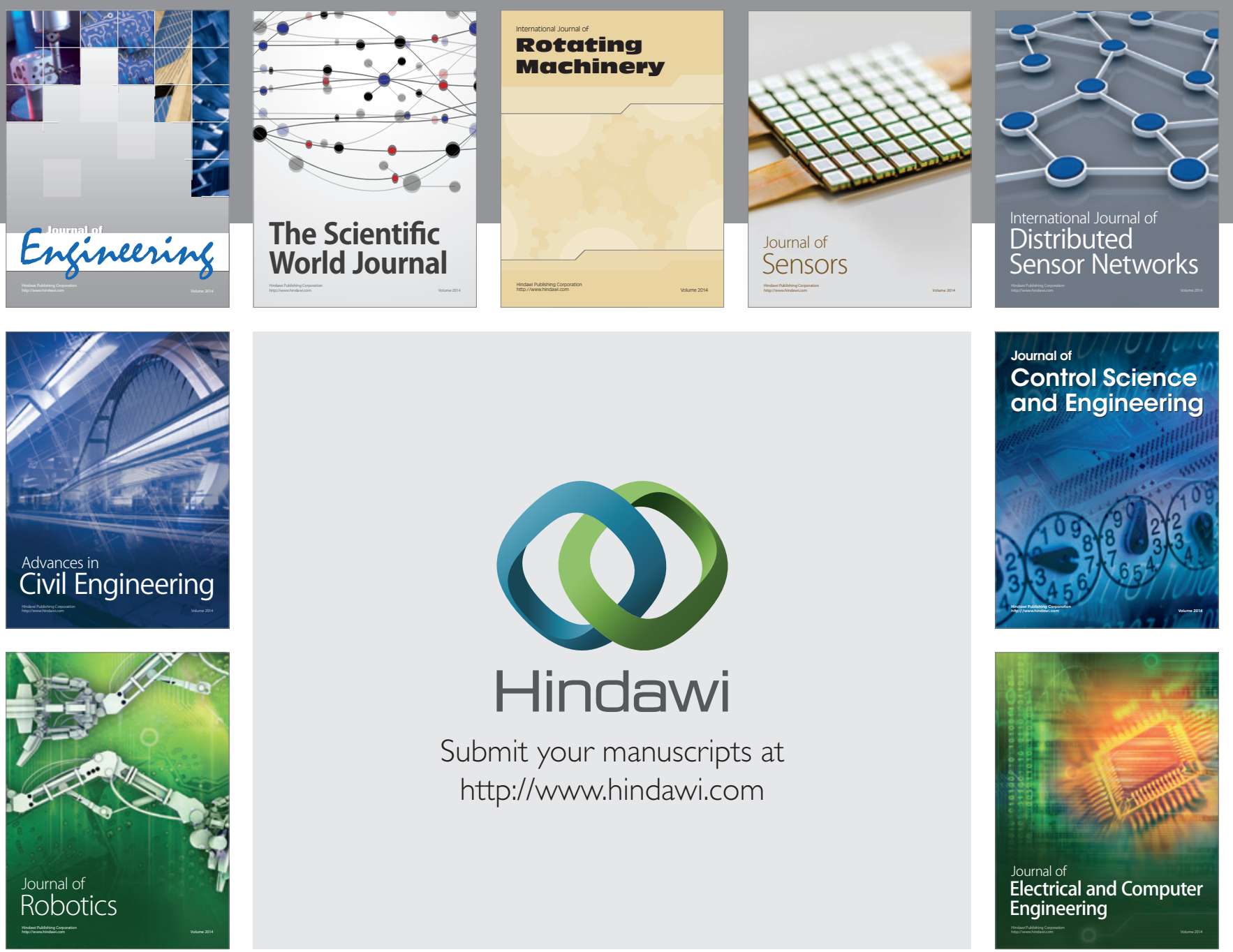

Submit your manuscripts at

http://www.hindawi.com
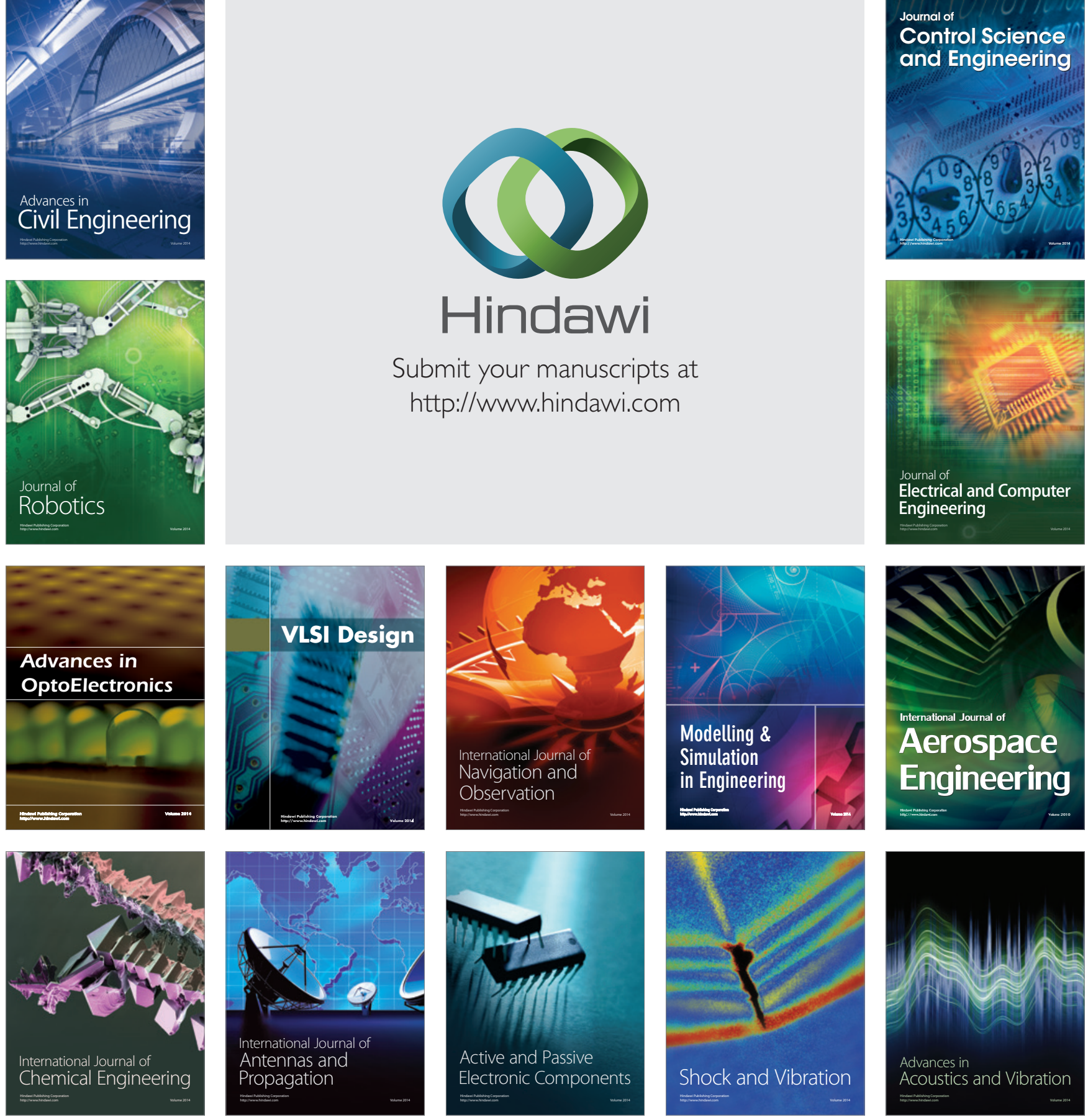